\title{
¿CÓMO PLANTEAR LAS VARIABLES DE UNA INVESTIGACIÓN?: OPERACIONALIZACIÓN DE LAS VARIABLES.
}

\section{HOW TO PROPOSE THE VARIABLES OF AN INVESTIGATION?: OPERATIONALIZATION OF THE VARIABLES.}

\author{
Villavicencio-Caparó Ebingen $^{1}{ }^{*}$, Torracchi-Carrasco Esteban ${ }^{1}$, Pariona-Minaya Maria del Carmen ${ }^{1}$,Alvear-Córdova \\ María Cristina ${ }^{1}$ \\ ${ }^{1}$ Docente de la Carrera de Odontología, Universidad Católica de Cuenca. \\ evillavicencioc@ucacue.edu.ec
}

\begin{abstract}
Resumen
El objetivo de este trabajo, es realizar una contribución didáctica docente que revise los contenidos necesarios y suficientes para la ejecución del planteamiento de las variables en la elaboración de un proyecto de investigación. Se explican los pasos para la construcción de una tabla de operacionalización de variables, desarrolla contenidos y ejemplos con casos concretos aplicados en odontología y profesiones afines.
\end{abstract}

Palabras clave: Indicadores de Salud, Indicadores (Estadística), Tesis Académicas.

\begin{abstract}
The aim of this article is to guide our fellow teachers on how to properly present the variables when preparing a research proposal. It contains some important definitions and several examples taken from the dentistry field that help understanding operationalization of variables. The aim of this paper, is to make a contribution didactic teacher to review the necessary and sufficient for the implementation of the approach of the variables in the development of a research project contents. Develops content and examples that help understanding the operationalization of the variables, concrete cases related to the field of dentistry.
\end{abstract}

Key words: Health Status Indicators, Indicators (Statistics), Academic Dissertations .

\section{INTRODUCCIÓN}

Las características fundamentales del método científico son la validez (capacidad de medir lo que se plantea medir) y la confiabilidad (capacidad de repetir una medida en las mismas condiciones). ${ }^{1}$ Plantear adecuadamente las variables ayuda a garantizar la validez y confiabilidad de un estudio. Las variables son características medibles u observables de las unidades de estudio y que pueden asumir distintos valores. ${ }^{2,3}$ Son el eje central de la investigación del enfoque cuantitativo, debido a que traducen la información que obtiene el investigador en valores, los cuales pueden ser de tipo cuantitativo (expresados en números susceptibles de ser empleados en operaciones aritméticas) o cualitativo (expresados en categorías o características). ${ }^{4}$ Existe discrepancia entre las cátedras de investigación de diferentes universidades, respecto a la ubicación del cuadro de la operacionalización de variables en el contexto de un proyecto de investigación (o tesis): algunas abogan por presentarlo en el Planteamiento Teórico del proyecto de investigación, mientras que otras proponen ubicarlo en el capítulo de Materiales y Métodos. En la Cátedra de Investigación Científica de la carrera de Odontología de la Universidad Católica de Cuenca, creemos que este cuadro debe ser parte del planteamiento teórico, debido a que aclara la forma en la que se plantea el problema (relación de variables), el rol que desempeñan las variables y la definición teórica de cada una de ellas. Este trabajo fue motivado por la gran cantidad de veces que hemos observado confusiones de términos en los proyectos de tesis de nuestros estudiantes de pregrado (por ejemplo: dimensión-indicador, 
datos-escala, etc). De forma tal que el objetivo del presente trabajo es contribuir con los docentes de investigación científica en el área de Odontología y carreras afines, para orientar correctamente la elaboración de proyectos de investigación.

\section{ESTADO DEL ARTE}

Operacionalizar las variables significa presentarlas en una tabla y descomponerlas en sus partes constitutivas para facilitar su comprensión inequívoca. ${ }^{5}$ La función de la operacionalización es presentar al lector la manera en la que se han conceptualizado y operativizado las variables, así como la forma en la que se van a tratar desde el punto de vista estadístico. Por ese motivo se presentan las variables de un estudio en una tabla de doble entrada, en la que las filas contienen las variables del estudio y las columnas las características de cada una de ellas. La operacionalización cumple con la función metodológica de orientar la definición de los objetivos del proyecto de investigación. Una variante de la operacionalización de las variables es la denominada matriz de consistencia, que es un esquema traducido a tabla, donde se explicitan de manera resumida todas las partes del proyecto de investigación.

Desde nuestro punto de vista, las características (columnas) que deben estar incluidas como mínimo en una tabla correcta de operacionalización de variables son:

1) VARIABLE: Contiene el nombre de la variable y el rol que desempeña en el estudio. Probablemente se genera confusión respecto a esta columna porque para la Organización Mundial de la Salud, los indicadores de salud ( moratlidad, morbilidad,etc.) son variables y se consideran como instrumentos que intentan objetivar los cambios en la gestión de salud ${ }^{1}{ }^{6}$ Nosotros sugerimos diferenciar de manera clara el concepto de variable y de indicador, como se verá en el apartado cinco.

Respecto al rol de la variable tenemos que esta puede ser variable única, en el caso de estudios descriptivos o comparativos. Aparecen las variables independiente y dependiente, en estudios relacionales y explicativos. Se consideran los roles de variable estímulo y variable respuesta en el caso de estudios experimentales.

Solo en el caso en que el diseño es poco eficiente, lo que se debe a que hay alguna característica de las unidades de estudio que, si bien logramos identificar, no hemos podido controlar, apareceran co-variables o variables intervinientes,. y esto se debe a que hay alguna característica de las unidades de estudio que, si bien logramos identificar, no hemos podido controlar. Conviene reportar honestamente esta situación, en la discusión, mencionándolo como una limitación del estudio para evitar que el mismo sea objetado por no haber notado su existencia.

A partir del desarrollo de la estadística factorial multivariante $^{7}$ en los diseños experimentales, las variables se clasifican en: *Observadas.- Endógenas ( Dependientes) y Exógenas (Independientes) *Latentes.- que solo se pueden identificar a partir del análisis estadístico factorial.

Un deslinde terminológico importante proviene de las ciencias sociales. En efecto, según algunos autores, se debe diferenciar a las variables intervinientes de las variables latentes ${ }^{7}$, a pesar de que ambas son variables confusoras: la primera es detectada a priori (en el diseño del estudio) y la segunda a posteriori (en el análisis estadístico).

En cuanto a variables intervinientes es importante diferenciar también a las variables mediadoras (identifican por qué y cómo los tratamientos tienen efectos) de las variables moderadoras (identifican en quién y bajo qué circunstancias los tratamientos tienen diferentes efectos.) $)^{8}$

En la Tabla 1 se presentan ocho variables como ejemplo. Se aprecia que el fenómeno caries puede ser operacionalizado en una misma investigación, de dos formas distintas. Siguiendo esta lógica, para cada trabajo el investigador puede operacionalizar de distinta manera una misma variable, y esto va a depender del objetivo y la intención que se tenga al momento de recoger el dato de la variable. A diferencia del caso anterior, otras variables que se presentan en la Tabla 1, presentan múltiples dimensiones. Estas variables son: Enfermedades periodontales (dos dimensiones), Maloclusión (nueve dimensiones), Higiene Bucal (dos dimensiones). Estas son consideradas variables multidimensionales.

2) DEFINICIÓN TEÓRICA: Esta columna presenta el concepto que se encuentra en los libros para definir la variable en cuestión. Es la representación mental que tenemos sobre la variable. (Ej. La caries dental es una enfermedad multifactorial que produce la desmineralización de los tejidos duros del diente).

3) DEFINICIÓN OPERATIVA: Son las características que se esperan observar en las unidades de estudio para evaluar la variable. Es la manera cómo el investigador capta los datos del objeto; dicho de otra forma, es la manera cómo los sentidos perciben la variable. (Ej. Para el índice CPOD, se considera que la caries está presente cuando se encuentra una cavitación en cuyo fondo el tejido está reblandecido.)

4) DIMENSIONES: Son las partes constitutivas de una variable. En Latinoamérica algunas facultades erróneamente usan este término como sinónimo de indicador de la variable. Las dimensiones se usan cuando la variable es un constructo (está constituida por más de una característica, por ejemplo la variable maloclusión 


\begin{tabular}{|c|c|c|c|c|c|c|c|c|}
\hline $\begin{array}{l}\begin{array}{c}\text { VARIABLE } \\
\text { (ROL) }\end{array} \\
\end{array}$ & DEF. TEORICA & $\begin{array}{c}\text { DEF. } \\
\text { OPERATIVA }\end{array}$ & DIMENSIONES & INDICADOR & $\begin{array}{c}\text { TIPO } \\
\text { ESTADISTICo }\end{array}$ & ESCALA & DATO & INSTRUMENTO \\
\hline \multirow{3}{*}{$\begin{array}{l}\text { Experiencia de } \\
\text { caries } \\
\text { (Dependiente) }\end{array}$} & \multirow{3}{*}{$\begin{array}{l}\text { Evidencia de las secuelas } \\
\text { de la caries dental en } \\
\text { dentición permanente. }\end{array}$} & \multirow{3}{*}{$\begin{array}{l}\text { Cantidad de } \\
\text { piezas } \\
\text { cariadas, } \\
\text { perdidas por } \\
\text { caries y } \\
\text { obturadas. } \\
\end{array}$} & Cariadas & \multirow{3}{*}{$\begin{array}{c}\text { CPOD de } \\
\text { Klein y Palmer }\end{array}$} & \multirow{3}{*}{$\begin{array}{l}\text { Cuantitativo } \\
\text { Discreto }\end{array}$} & \multirow{3}{*}{$\begin{array}{c}\mathrm{De} \\
\text { Intérvalo }\end{array}$} & \multirow{3}{*}{$\mathrm{De} 0$ a 32} & \multirow{3}{*}{ Odontograma } \\
\hline & & & Perdidas & & & & & \\
\hline & & & Obturadas & & & & & \\
\hline $\begin{array}{l}\text { Prevalencia de } \\
\text { Caries } \\
\text { (Dependiente) }\end{array}$ & $\begin{array}{l}\text { Proporción de personas } \\
\text { con presencia actual de } \\
\text { caries dental. }\end{array}$ & $\begin{array}{l}\text { Proporción de } \\
\text { personas con } \\
\text { presencia } \\
\text { actual de } \\
\text { caries dental. }\end{array}$ & & Porcentaje & $\begin{array}{l}\text { Cualitativo } \\
\text { Nominal }\end{array}$ & Nominal & $\begin{array}{l}\text { Sano } \\
\text { Enfermo }\end{array}$ & Odontograma \\
\hline \multirow[t]{2}{*}{$\begin{array}{l}\text { Enferedades } \\
\text { Periodontales } \\
\text { (Dependiente) }\end{array}$} & \multirow{2}{*}{$\begin{array}{c}\text { Enfermedad inflamatoria } \\
\text { asociada a acumulación } \\
\text { de placa dento bacteriana, } \\
\text { que afecta los tejidos de } \\
\text { soporte del diente. }\end{array}$} & \multirow{2}{*}{$\begin{array}{c}\text { Evidencia de } \\
\text { inflamación } \\
\text { gingival, } \\
\text { bolsas } \\
\text { periodontales } \\
\text { y/o pérdida de } \\
\text { función } \\
\text { periodontal. }\end{array}$} & $\begin{array}{l}\text { Gingivitis } \\
\text { (inflamación de la encia) }\end{array}$ & \multirow{2}{*}{\begin{tabular}{|c|} 
PI \\
Indice \\
Periodontal de \\
Russell
\end{tabular}} & \multirow[t]{2}{*}{$\begin{array}{l}\text { Cualitativo } \\
\text { Ordinal }\end{array}$} & \multirow[t]{2}{*}{ Ordinal } & \multirow{2}{*}{$\begin{array}{c}\text { *Encia sana } \\
(\text { de } 0 \text { a } 0,2) \\
* \text { Gingivitis simple } \\
(\text { de } 0,3 \text { a } 0,9) \\
* \text { Periodontitis inicial } \\
\text { (de } 1,0 \text { a } 1,9) \\
\text { *Pediodontitis establecida } \\
\text { (de } 2,0 \text { a } 5 \text { ) } \\
\text { *Periodontitis Terminal } \\
\text { (de } 5,0 \text { a 8) }\end{array}$} & \multirow[t]{2}{*}{ Periodontograma } \\
\hline & & & \begin{tabular}{|l} 
Periodontitis \\
(inflamación y/o \\
destrucción del tej. \\
Periodontal) \\
\end{tabular} & & & & & \\
\hline \multirow{9}{*}{$\begin{array}{c}\text { Maloclusión } \\
\text { (Interviniente) }\end{array}$} & \multirow{9}{*}{$\begin{array}{c}\text { Alteración en la relación } \\
\text { tridimensional adecuada } \\
\text { de las primeras molares } \\
\text { superiores e inferiores, o } \\
\text { que alteración de la linea } \\
\text { de oclusión } \\
\text { uniformemente curvada. }\end{array}$} & \multirow{9}{*}{\begin{tabular}{|c|} 
Presencia de \\
relación molar \\
alterada, \\
resalte, \\
sobrepase, \\
diastemas, \\
apiñamiento, \\
mordidas \\
cruzadas, \\
mordidas \\
abiertas y/o \\
falta de algún \\
diente.
\end{tabular}} & Llaves molares & \multirow{9}{*}{$\begin{array}{l}\text { Clasificación } \\
\text { de Angle. }\end{array}$} & \multirow{9}{*}{$\begin{array}{c}\text { Cualitativo } \\
\text { Nominal }\end{array}$} & \multirow{9}{*}{ Nominal } & \multirow{9}{*}{$\begin{array}{l}\text { Normoclusión } \\
\text { Maloclusión de clase I } \\
\text { Maloclusión de clase II-1 } \\
\text { Maloclusión de clase II-2 } \\
\text { Maloclusión de clase III }\end{array}$} & \multirow{9}{*}{$\begin{array}{l}\text { Ficha de } \\
\text { observación de la } \\
\text { Historia clínica } \\
\text { Ortodóntica. }\end{array}$} \\
\hline & & & Llaves caninas & & & & & \\
\hline & & & Sobre pase & & & & & \\
\hline & & & Resalte & & & & & \\
\hline & & & Diastemas & & & & & \\
\hline & & & Apiñamiento & & & & & \\
\hline & & & Mordidas cruzadas & & & & & \\
\hline & & & Mordidas abiertas & & & & & \\
\hline & & & Edentulismo & & & & & \\
\hline
\end{tabular}

Tabla 1. EJEMPLO DE OPERACIONALIZACIÓN DE VARIABLES EN INVESTIGACIÓN ODONTOLÓGICA

Tabla No1), por otra parte existen variables que se miden directamente, en ese caso carecen de dimensiones.(Ej. Prevalencia de caries Tabla No 1). Existen variables con nivel de organización más complejo, que necesitan ser descompuestas en dimensiones y estos a su vez en sub subdimensiones. Existen también casos de variables tan complejas que necesitan hasta cuatro niveles de desagregación; en este caso la variable se divide en dimensiones, estos en sub dimensiones de primer orden, de segundo orden y de tercer orden. Si planteamos una variable que en su interior tiene dos indicadores y estos no pueden unirse para dar un solo valor para la variable, es preferible manejar estas dos entidades como variables separadas, debido a que en la base de datos generarán dos columnas diferentes que nunca podrán ser resumidas en un solo dato, por lo tanto, se deberán reportar en dos tablas estadísticas diferentes. En el ejemplo de la tabla 1; el error frecuente es tener una sola variable denominada caries, con dos dimensiones (experiencia de caries y prevalencia de caries) que no se pueden conjugar en un solo dato, dado que una es de carácter cualitativo y la otra cuantitativo.

5) INDICADORES: Son expresiones matemáticas que nos permite medir variables compuestas. Un claro ejemplo de ello son algunos indicadores epidemiológicos en Odontología (Ej. CPOD, Índice de Russell, IHO-S, etc). ${ }^{9}$ En algunas universidades los indicadores son considerados las partes constitutivas de la variable, sobre todo si esta es un constructo. Sin embargo consideramos que esto es un error muy común, y que esta confusión debe ser esclarecida, al entender el concepto de dimensión de la variable(Algunas veces las dimensiones tienen su traducción matemática en un indicador, en otras oportunidades la dimensión de expresa directamente con el dato). Por ejemplo en la tabla 1 presentamos la variable "Enfermedades Periodontales" que tiene dos dimensiones, mientras que su indicador es el Índice de Russell (IP) ${ }^{10}$ el cual puede ser representado por una fórmula:

$n=\frac{\text { sumadecdigosdecadadiente }}{\text { sumadedientesexaminados }}$

6) TIPO ESTADÍSTICO DE LA VARIABLE: En esta columna se informa acerca de la naturaleza estadística de la variable, con la finalidad de orientar el plan de análisis de los datos. ${ }^{11}$ Por ejemplo, en el caso de la variable de tipo cualitativo "Prevalencia de Caries" presentada en la Tabla 1, se hace referencia a la presencia o ausencia de caries, y por lo tanto su reporte en el informe final se hará bajo la forma de frecuencias y porcentajes. Por otro lado, si esta misma enfermedad fuera operacionalizada como "Experiencia de caries", sería considerada de tipo cuantitativo y se referiría a la cantidad de piezas afectadas 


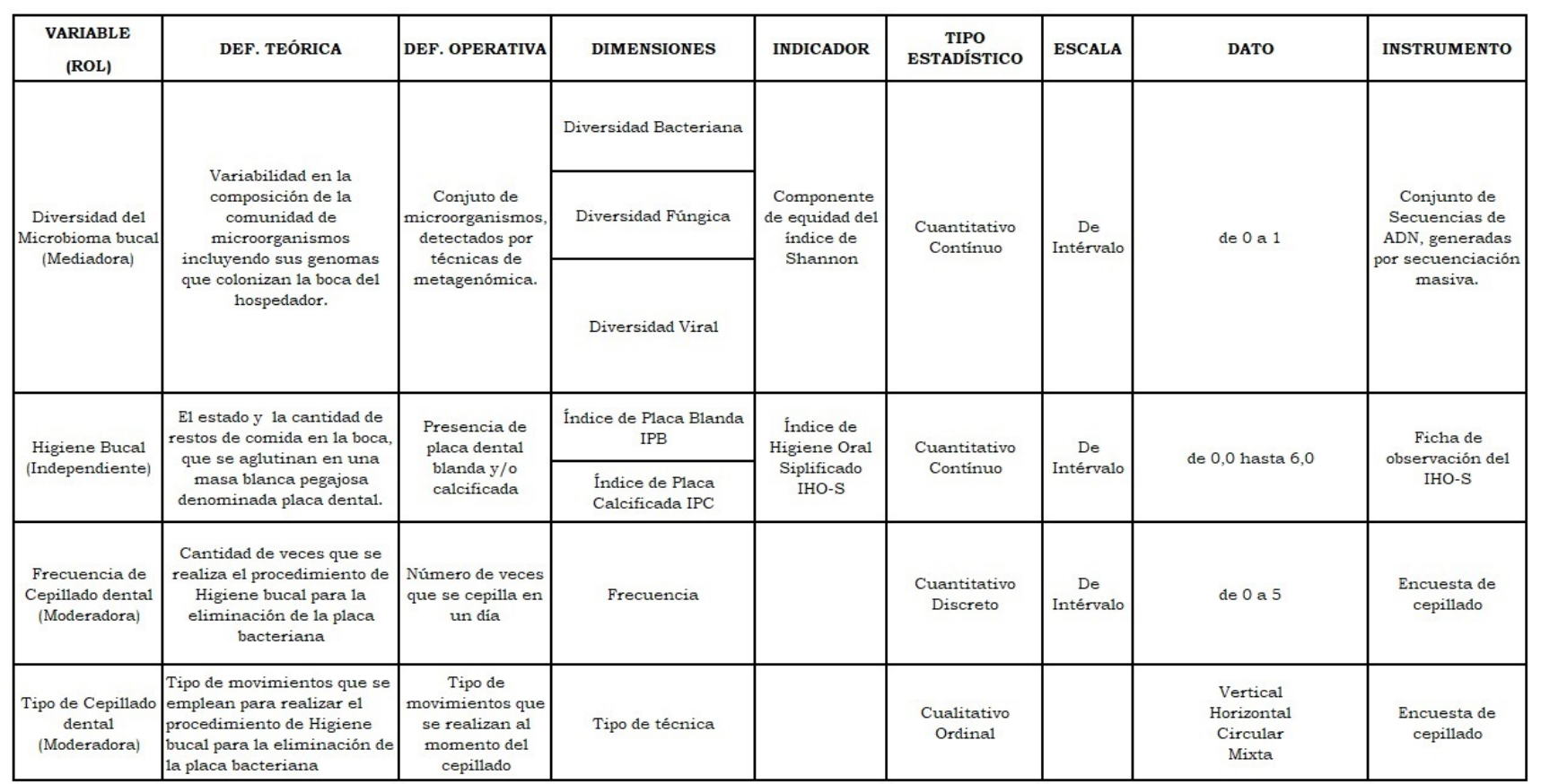

Tabla 2. EJEMPLO DE OPERACIONALIZACIÓN DE VARIABLES EN INVESTIGACIÓN ODONTOLÓGICA...continuación

por caries que tiene una persona. En este caso, el reporte en el informe final se haría en base a la presentación de promedios y desviación estándar. Esa es la importancia de esta columna, pues como ya se mencionó orienta el manejo estadístico de los datos.

Los cuatro tipos estadísticos de variables que existen son: ${ }^{12}$

- Cualitativa nominal.-cuando la variable tiene categorías que no pueden ser ordenadas.(Ej. Maloclusión)

- Cualitativa ordinal.- cuando la variable tiene categorías que pueden ser ordenadas por intensidad. (Ej. Índice periodontal de Russell)

- Cuantitativa discreta. - cuando la variable numérica no admite decimales.( Ej. Frecuencia de cepillado)

- Cuantitativa continua.- cuando la variable numérica admite decimales debido a que podemos mejorar la precisión de la medición en base a usar mejor tecnología para la medición (Ej. Higiene Bucal IHO-S).

Las variables cualitativas, deberán ser reportadas en tablas de frecuencias y porcentajes. ${ }^{3}$ Específicamente la variable nominal se grafica mediante diagramas de sectores y la variable ordinal mediante diagrama de barras separadas. ${ }^{3}$ Las variables cuantitativas deberán ser reportadas preferentemente en tablas que contienen estadísticos de Medidas de tendencia central, Medidas de dispersión, Medidas de Posición y Medidas de Forma. La manera adecuada de graficarlas es mediante el Histograma de frecuencia o el diagrama de caja y bigote. ${ }^{13}$

7) ESCALA: Existen cuatro tipos de escalas, se menciona el tipo al que pertenece porque tiene implicancias en la forma de presentar y resumir la información. Las escalas son: ${ }^{14}$

- Ordinal. - coincide con el tipo de variable

- Nominal. - también coincide con el tipo de variable

- De Razón. - Pertenece a variables cuantitativas donde el cero asume un valor absoluto, por lo tanto, no se admite como un valor que pueda tener esta variable.

- De intérvalo. - Pertenece a variables cuantitativas donde el cero asume un valor arbitrario, por lo tanto, puede existir el cero e inclusive valores negativos en la escala.

Es un error frecuente presentar en esta columna los datos que asume la variable como si esa fuera la escala, note que la columna dato, se ubica al lado de la variable escala en la Tabla No 1.

8) DATO(Valores): Esta columna informa acerca de los valores posibles de la variable. Tiene la finalidad de restringir valores que se consignarán en la base de datos, en proyección al control de calidad que se realizará previo al análisis de datos. ${ }^{15}$ Es muy útil para hacer el control de calidad de las bases de datos y eliminar los valores erróneos. Por este motivo, se recomienda 
obtener aproximadamente un 15\% adicional de datos, 14 Spiegel M. Estadística. 4th ed. México D.F.: Mc Graw Hill; para contrarrestar posibles pérdidas de información. 2000.

9) INSTRUMENTO: Consideramos que esta última colum- 15 Friedman L, Furberg C, DeMets D. Fundamentals of Clinina debe incluirse en la operacionalización para brindar información sobre el instrumento documental con el que se recogerán los datos. En textos clásicos de investigación científica solo se consideran tres tipos de instrumentos, a saber: la ficha de observación, la encuesta y la entrevista. Sin embargo, desde nuestro punto de vista y considerando los avances tecnológicos, esta clasificación debe ser revisada. En la Tabla 1 mostramos que en la actualidad hay instrumentos de obtención de datos que escapan a la clasificación antes mencionada. Ejemplo de ello es el caso del conjunto de secuencias de ADN que constituyen la información básica para estudios de ecología microbiana oral, que jamás necesitará una ficha de observación adicional y que funciona directamente como base de datos. ${ }^{7}$

\section{Referencias}

1 Organización Panamericana de la Salud. indicadores de Salud: Elementos Básicos para el Análisis de la Sitación de Salud. Boletín Epidemiológico / OPS. 2001; 22(4): p. 1-5.

2 Villavicencio-Caparó. Auditoría de la Investigación Científica Biomédica Arequipa; 2016.

3 Rius-Diaz F, Barón-López FJ. Bioestadística Madrid: Paraninfo; 2009.

4 Bunge M. La investigación científica Barcelona: Siglo XXI editores; 2004.

5 Tafur-Portilla R, Izaguirre-Sotomayor M. Cómo Hacer Un Proyecto De Investigación. In ; 2015; Medellín: Alfaomega.

6 Dawson B, Trapp R. Bioestadística médica Mexico D.F.: Manual moderno.

7 Quinn G. Expermental Design and Data Analysis for Biologists. In ; 2004; Edimburg United Kingdom: Cambridge.

8 Baron R, Kenny D. The moderator-mediator variable distinction in Social Phycological research: Conceptual, Strategic, and Statistical Considerations. 1986; 51(6): p. 11731182.

9 Sociedad Española de Periodoncia y Osteointegración. 1er Workshop Ibérico Control de placa e higiene bucodental. In Sanz M, editor. ; 2003; Madrid: Sociedad Española de Periodoncia y Osteointegración.

10 Cuenca-Sala E, Pilar BG. Odontología preventiva y comunitaria. Principios, métodos y aplicaciones.: Elsevier Masson; 2013.

11 Spiekerman C. Lecture Slides. Biostatistics in Dentistry Summer 2015. In ; 2015; Seattle: University of Washingtong.

12 Selvin S. A Biostatistics Toolbox for Data Análysis California: Cambridge; 2015.

13 Wayne WD. Bioestadística: Base para el análisis de las ciencias de la salud Mexico D.F.: Limusa; 2002. 
